\title{
Adatom Diffusion at GaN (0001) and (0001) Surfaces
}

\author{
Tosja Zywietz, Jörg Neugebauer, Matthias Scheffler \\ Fritz-Haber-Institut der Max-Planck-Gesellschaft, Faradayweg 4-6, D-14195 Berlin, Germany
}

(February 1, 2008)

\begin{abstract}
The diffusion of $\mathrm{Ga}$ and $\mathrm{N}$ adatoms has been studied for the technologically relevant wurtzite $(000 \overline{1})$ and (0001) surfaces employing density-functional theory. Our calculations reveal a very different diffusivity for $\mathrm{Ga}$ and $\mathrm{N}$ adatoms on the equilibrium surfaces: While Ga is very mobile at typical growth temperatures, the diffusion of $\mathrm{N}$ is by orders of magnitudes slower. These results give a very detailed insight of how and under which growth conditions $\mathrm{N}$ adatoms can be stabilized and efficiently incorporated at the surface. We further find that the presence of excess $\mathrm{N}$ strongly increases the Ga diffusion barrier and discuss the consequences for the growth of GaN.
\end{abstract}

Recently, great progress in fabricating highly efficient GaN-based devices has been achieved [1,2]. Nevertheless, there are still substantial problems concerning growth optimization and insight into the fundamental mechanisms is rather shallow. Investigations of molecular beam epitaxy (MBE) growth have shown that the film structure and morphology are very sensitive to the III/V ratio [3, 4 . GaN films grown under more N-rich conditions are rough and faceted while going towards more Ga-rich conditions a smoother surface morphology and better film quality are obtained [3].

In order to improve growth in a systematic way it is essential to understand the underlying kinetic processes such as adsorption, desorption, and surface diffusion. In particular, adatom diffusion on surfaces is considered to be a key parameter controlling the growth rate, the material quality, and the surface morphology. Experimentally, an analysis of surface diffusion is difficult: So far only effective diffusion barriers have been obtained for $\mathrm{GaN}$ [回.5]. It is also not clear whether the cation or anion surface diffusion is the rate limiting process [4]. A problem in growing $\mathrm{GaN}$ is also the high $\mathrm{N}$ vapor pressure and considerable efforts have been made to enhance the $\mathrm{N}$ incorporation. However, the mechanism by which $\mathrm{N}$ is incorporated at the surface could not be identified. Theoretically, only few studies to describe GaN growth have been reported, which were either based on thermodynamic models [6] or Monte Carlo simulations [7]. These approaches are useful to model growth on a mesoscopic scale. However, the microscopic meaning of the effective parameters and the underlying microscopic processes remain unclear. In order to identify the fundamental growth mechanisms, but also to improve the above mentioned methods, the correct microscopic parameters are needed [8].

We have therefore performed a comprehensive and detailed study of the migration and energetics of Ga and $\mathrm{N}$ adatoms on GaN surfaces, employing totalenergy density-functional theory calculations with $a b$ initio pseudopotentials. We will focus on the two technolog- ically relevant orientations for wurtzite GaN, the (0001) and the $(000 \overline{1})$ surfaces. The adatom substrate system is modeled by supercell geometries with at least 9 layers of GaN, a 14 bohr vacuum region and $(2 \times 2)$ periodicity. One side of the slab is passivated by fractional pseudohydrogen. Tests revealed that migration paths and diffusion barriers are not significantly affected by the Ga $3 d$ electrons and that it is sufficient to treat them within the non-linear core correction (NLCC) 99. Details of the calculations are discussed elsewhere 10,11].

The energetically stable reconstructions for polar GaN surfaces have been recently identified for the cubic (001) 112] and the wurtzite (0001) and (000) orientations [13]. These studies revealed a surprising feature not observed for "traditional" III-V semiconductor surfaces: Independent of the chemical environment (Ga- or N-rich) polar GaN surfaces turn out to be always cation stabilized with no $\mathrm{N}$ atoms in or on-top of the surface layer. The only known exception so far is a $\mathrm{N}(2 \times 2)$ adatom structure on the (0001) surface found to be stable under very $\mathrm{N}$ rich conditions [13]. In order to study adatom diffusion on the thermodynamical stable surfaces we will therefore focus on the two structures shown in Fig. 1 which both are characterized by a complete Ga surface layer.

We first calculated the potential energy surface (PES) for Ga and $\mathrm{N}$ adatoms on the (0001) surface, giving immediate insight into stable sites, migration paths, and diffusion barriers. The potential energy surface $E_{\text {tot }}\left(\mathbf{R}_{\|}^{\text {ad }}\right)$ is calculated by fixing the adatom laterally at different positions $\mathbf{R}_{\|}^{\text {ad }}$ and allowing all other atoms and the adsorbate height to relax. A two-dimensional (2D) cut through the resulting potential energy surfaces for $\mathrm{Ga}$ and $\mathrm{N}$ adatoms along the energetically lowest path is shown in Fig. 2. For Ga adatoms we find two adsorption sites: fcc and hcp (see Fig. 1), which are degenerate in energy within the accuracy of our method. Fig. 2 also reveals that the energetically lowest transition site [corresponding barrier: $(0.4 \mathrm{eV})]$ is the bridge position; and the migration over the on-top position is energetically very unfavorable. Actually, an analysis of the complete PES 
showed that the on-top position is the global maximum.

For $\mathrm{N}$ adatoms a qualitatively very different PES results (Fig. 2, dashed line): While the fcc position is the energetically lowest binding site, the hcp position is significantly higher in energy $(1.4 \mathrm{eV})$. Therefore, to hop from one fcc site to the next, the $\mathrm{N}$ adatom diffuses along the bridge position over the hcp site which is the transition site leading to a barrier of $1.4 \mathrm{eV}$. The large energy difference between the fcc and hcp sites can be understood by analyzing the atomic structure of the surface: At the hcp position, the $\mathrm{N}$ adatom experiences directly underneath a $\mathrm{N}$ atom in the second layer. Since GaN is a partly ionic material this leads to electrostatic repulsion [14.

When analyzing adsorption sites it should be kept in mind that the on-top position is the epitaxial site for $\mathrm{N}$ adatoms on the (0001) surface, i.e., for high coverages this site must be a global minimum. From Fig. 2 we find this site to be energetically highly unfavorable for an isolated $\mathrm{N}$ adatom (i.e. for low coverages). Therefore, when going from low to high adatom coverages the $\mathrm{N}$ adatoms have to shift from the fcc site (stable at low coverages) to the on-top position (stable at high coverages). Since this process is kinetically hindered, the initial adsorption at the fcc position might be a potential source for stacking faults.

We have also studied the diffusion on the $(000 \overline{1})$ surface, which is characterized by a very different atomic geometry where each surface atom has three dangling bonds compared to only one at the (0001) surface (see Fig. 1). For Ga adatoms the PES (see Fig. 3) shows very similar features as found on the (0001) surface: The fcc and hcp sites are energetically degenerate, the diffusion transition state is at the bridge site with an again very low energy barrier $(0.2 \mathrm{eV})$.

For $\mathrm{N}$ adatoms we find a qualitative different behavior compared to $\mathrm{N}$ atoms on the (0001) surface (see Fig. 3): (i) N adatoms exhibit the same binding sites than Ga adatoms and (ii) the diffusion barrier is significantly lower $[0.9 \mathrm{eV}$ compared to $1.4 \mathrm{eV}$ on (0001)]. The lower diffusion barrier can be understood in terms of the very different character of the chemical bonds on the (0001) and (0001) surfaces. On the (0001) surface (see Fig. 1) the localized and strongly directed $s p_{z}$ orbitals favor the formation of strong Ga-N bonds giving low coordinated sites, as e.g. the bridge position, a rather high energy. On the other hand, the (0001) surface is characterized by metallic bonds between the surface atoms: the adsorbatesubstrate interaction is hence significantly weaker resulting in lower diffusion barriers.

An important consequence of our results is that $\mathrm{Ga}$ adatoms have a significantly lower diffusion barrier than $\mathrm{N}$ adatoms for both orientations: Ga adatoms will be orders of magnitude more mobile than $\mathrm{N}$ adatoms at typical growth temperatures. The low diffusion barrier is a direct consequence of the fact, that for $\mathrm{GaN}$ equilibrium surfaces are Ga stabilized. Thus, for Ga atoms the adsorbate-substrate interaction is predominantly realized by delocalized metallic Ga-Ga bonds. Since Ga-bulk melts already slightly above room temperature $\left(T_{\text {melt }}=30^{\circ} \mathrm{C}\right)$, the Ga-Ga bonds are weak and the adatoms behave almost like a liquid film on the surface. A similar effect has not been reported at "traditional" III/V semiconductor surfaces like e.g. GaAs where the surfaces do not exhibit a metallic like character. The diffusion barrier on these surfaces is thus mainly characterized by breaking strong cation-anion bonds. As a consequence a significantly higher Ga diffusion barrier is found on these surfaces, e.g., for the polar GaAs (111) surface the barrier is $0.9 \mathrm{eV} 15$.

The significantly larger diffusion barrier for $\mathrm{N}$ adatoms compared to $\mathrm{Ga}$ adatoms on equilibrium surfaces has an important consequence: Although $\mathrm{N}$ adatoms on these surfaces are thermodynamically unstable against evaporation as $\mathrm{N}_{2}$-molecules even under N-rich conditions [12], they can be kinetically stabilized at the surface. In order to evaporate, two $\mathrm{N}$ atoms have to form a $\mathrm{N}_{2}$ molecule. Since migration of $\mathrm{N}$ adatoms is a highly activated process (but necessary to form molecules), the desorption rate may become smaller than the adsorption rate. Consequently, extended regions in which the surface is primarily covered by $\mathrm{N}$ atoms may be formed. These $N$ adatoms are likely to influence the migration path and the diffusion barrier of $\mathrm{Ga}$ adatoms. We have therefore studied the diffusion of Ga adatoms on the N-terminated $(0001)$ and $(000 \overline{1})$ surfaces which can be considered as "extreme" cases of $\mathrm{N}$ coverage. The results are shown in Fig 4. The energetically favored binding sites are located at the fcc and hcp positions and the transition site is the bridge position. For both surface orientations the diffusion barrier of Ga adatoms is strongly affected: At (0001) the migration barrier increases from 0.4 to $1.8 \mathrm{eV}$ while at $(000 \overline{1})$ it increases from 0.2 to $1.0 \mathrm{eV}$. We therefore conclude that excess $\mathrm{N}$ at the surface, which can be formed under N-rich conditions, significantly reduces the mobility of $\mathrm{Ga}$ adatoms. The reason is the formation of strong Ga-N bonds, which have to be broken during the migration of the adatoms.

The reduction in surface diffusivity when going towards more $\mathrm{N}$-rich conditions is consistent with a recent very detailed MBE growth study by Tarsa et al. [3]. We note however, that a direct comparison between our calculated diffusion barriers and experimentally derived barriers is by no means straightforward. Experimentally, effective diffusion parameters are commonly derived which average over a large area including not only the clean surface but also steps, impurities, dislocation etc. Since the effective diffusion barrier is usually dominated by the process with the highest barrier, our calculated value (which is for the clean surface) is a lower limit of the effective diffusion barrier.

The very different mobilities of $\mathrm{Ga}$ and $\mathrm{N}$ adatoms 
and the formation of excess $\mathrm{N}$ have important consequences for the growth of GaN. In the Ga-rich regime where the amount of excess $\mathrm{N}$ on the surface is small, the Ga adatoms are highly mobile and a step-flow mode resulting in $2 \mathrm{D}$ growth is expected. As a consequence, the surface morphology should be improved and a low density of stacking faults is expected. Further, if excess $\mathrm{Ga}$ adatoms are present on the surface (as expected for Ga-rich growth conditions), $\mathrm{N}$ adatoms can be efficiently incorporated: The probability that fast moving Ga adatoms capture $\mathrm{N}$ atoms is much higher than the other process where $\mathrm{N}$ atoms form molecules and desorb from the surface. However, at the N-terminated surfaces our results show a roughly five times higher diffusion barrier, indicating that the Ga diffusion length is significantly shorter under N-rich conditions. Once the diffusion length is shorter than the mean distance between the binding sites a statistical roughening of the surface can be expected. Furthermore, the adatoms might be "trapped" at sites not corresponding to the ideal bulk positions. For example, if a Ga atom is "trapped" at the fcc position and does not hop to the wurtzite hcp site, a fcc nucleation center is formed. Thus, the higher adatom mobility under more Ga-rich conditions will also significantly reduce the density of stacking faults. Based on a detailed analysis of the adatom kinetics we expect therefore slightly Ga-rich conditions to be optimal for the growth of GaN. We note however, that while going towards extreme Ga-rich conditions might further improve the growth morphology, it will also enhance the impurity incorporation [9] and result in high background carrier concentrations.

We gratefully acknowledge financial support from the BMBF, the Fond der Chemischen Industrie (T.Z.), and the Deutsche Forschungsgemeinschaft (J.N.).

[1] S. Nakamura, T. Mukai, and M. Senoh, Appl. Phys. Lett. 64, 1687 (1994).

[2] S. Nakamura et al., Appl. Phys. Lett. 70, 868 (1997).

[3] E. J. Tarsa et al., J. Appl. Phys. 82, 5472 (1997).

[4] H. Yang et al., Appl. Phys. Lett. 68, 244 (1996).

[5] E. R. Weber, private com.

[6] S. Y. Karpov and Y. N. Makarov and M. S. Ramm, MRS Internet J. Nitride Semicond. Res. 2, 45 (1997).

[7] K. Wang and J. Singh and D. Pavlidis, J. Appl. Phys. 76, 3502 (1994).

[8] P. Ruggerone and C. Ratsch and M. Scheffler, Growth and Properties of Ultrathin Epitaxial Layers, (Elsevier Science, Amsterdam, 1997), Vol. 8.

[9] T. Zywietz and J. Neugebauer and M. Scheffler, to be published.

[10] R. Stumpf and M. Scheffler,Comp. Phys. Commun. 79,
447 (1994).

[11] J. Neugebauer and C. G. Van de Walle, Proc. Mater. Res. Soc. Symp. 408, 43 (1995).

[12] J. Neugebauer et al., Phys. Rev. Lett. 80, 3097 (1998).

[13] A. R. Smith et al., Phys. Rev. Lett. 79, 3934 (1997).

[14] K. Rapcewicz and M. B. Nardelli and J. Bernholc, Phys. Rev. B 56, R12753 (1997).

[15] A. Kley and P. Ruggerone and M. Scheffler, Phys. Rev. Lett. 79, 5278 (1997).

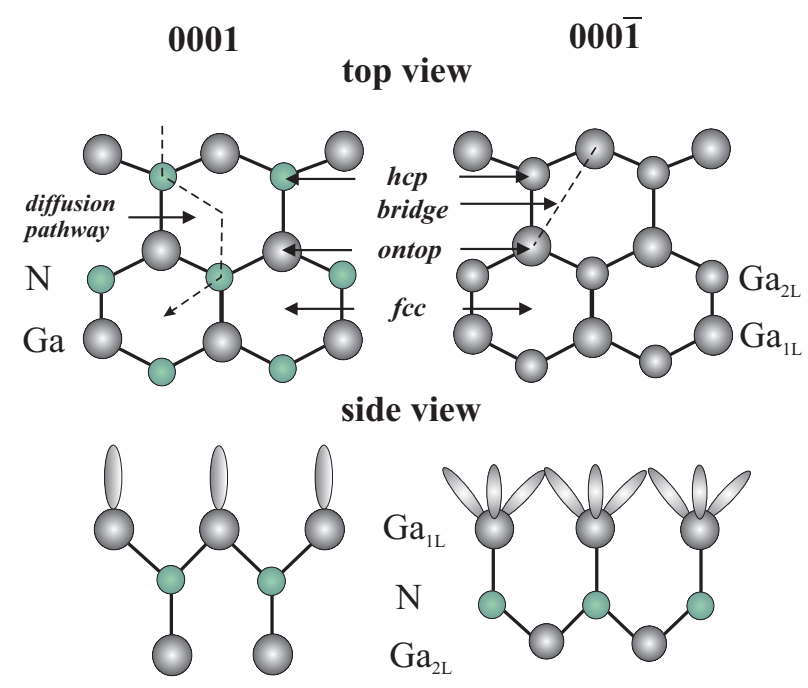

FIG. 1. Atomic structures (top and side view) for the (0001) and (0001) Ga terminated surfaces. The dashed line in the top left marks the diffusion pathway corresponding to Fig. 2 and Fig. 3. $G a_{1 L}$ marks a Ga-atom in the first layer, $G a_{2 L}$ in the second.

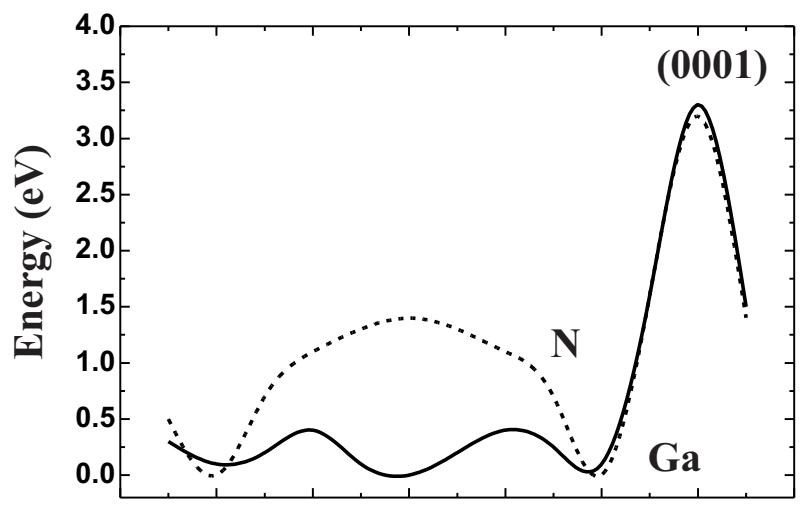

fcc bridge hep bridge fcc on-top

FIG. 2. Total energy (in eV) for Ga-adatom (solid line) and a N-adatom (dashed line) at the Ga-terminated (0001) surface. The energy zero corresponds to the energetically lowest adsorption site. 


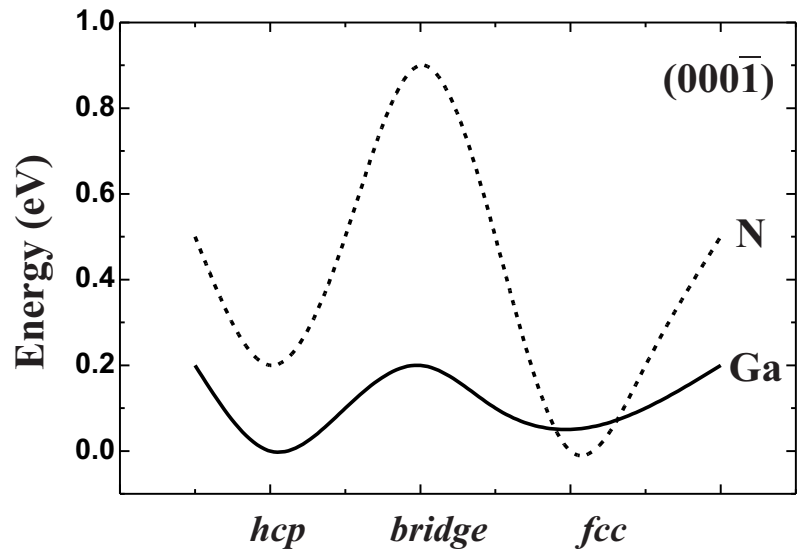

FIG. 3. Total energy (in eV) for a Ga-adatom and a $\mathrm{N}$-adatom at the Ga-terminated (0001) surface. The energy zero corresponds to the energetically lowest adsorption site.

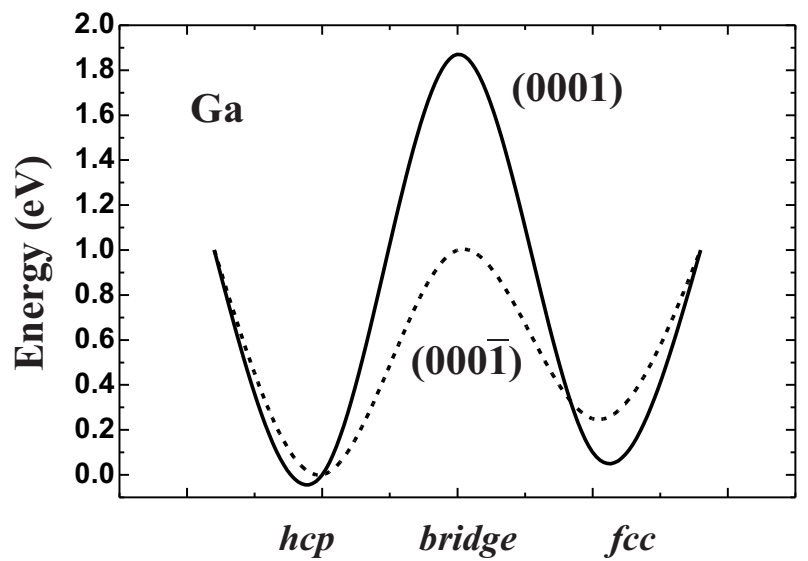

FIG. 4. Total energy (in eV) for Ga-adatoms at the $\mathrm{N}$-terminated (0001, solid line) and the N-terminated (000 $\overline{1}$, dashed line) surface. The energy zero corresponds to the energetically lowest adsorption site. 